\title{
Experimental demonstration that observers produce unbiased estimates of reduction lightness in transparent surfaces
}

\author{
SERGIO CESARE MASIN and ADA QUARTA \\ University of Padua, Padua, Italy
}

\begin{abstract}
The perceptual properties of a transparent surface may be evaluated under a normal or an analytical attitude. Under the normal attitude, we perceive two overlapping surfaces: one transparent and one a background. Under the analytical attitude, we perceptually isolate single regions in the transparent surface. The methodological problem of whether observers are able to maintain an analytical attitude when asked to rate the single lightness of an isolated region has been faced here. The results show that observers have this ability. Moreover, the results indicate that the lightness estimated under the analytical attitude is entirely independent of the lightnesses of the two overlapping surfaces perceived under the normal attitude.
\end{abstract}

Current theories of phenomenal transparency (Beck, Prazdny, \& Ivry, 1983; Metelli, 1982, 1983) assume that the percept is the result of an integration of local chromatic information. For example, Metelli's theory states that the apparent degree of transparency, $\alpha$, of the rectangle in Figure 1 is given by the equation $\alpha=$ $(p-q) /(a-b)$, where $a$ and $b$ are the lightnesses and $p$ and $\mathrm{q}$ are the reduction lightnesses of the regions $\mathrm{A}, \mathrm{B}, \mathrm{P}$, and $Q$ as indicated in Figure 2a. (The reduction lightness of region $\mathrm{P}$, or $\mathrm{Q}$, is the lightness of that region when transparency is not perceived. ${ }^{1}$ ) Dependent on the theoretical context, the lightnesses $a, b, p$, and $q$, or the differences $(p-q)$ and $(a-b)$, may be conceived as the local chromatic information. Obtaining estimates of local information is a prerequisite for testing the theories.

Lightnesses, or differences between lightnesses, may be estimated, for example, by the numerical rating method. However, there is no proof that the estimates of $\mathrm{p}$ and $\mathrm{q}$, or of their difference, are not influenced by the transparency phenomenon. In other words, in looking at a transparent surface (e.g., the rectangle in Figure 1), we may adopt a normal or an analytical attitude. Under the normal attitude, we perceive a single transparent surface. Under the analytical attitude, we isolate the various regions in the field. When a normal attitude is assumed, two colors are perceived in region $\mathrm{P}$, or in $\mathrm{Q}$ (that is, the color of the transparent surface and the color of the part of the background seen through the transparent surface). It might well be that the observer, under the analytical attitude, is not able to perfectly isolate region $\mathrm{P}$ and, consequently, when asked to rate $p$, makes some compromise between the two colors perceived under the normal attitude.

The experiment reported here had the purpose of solving the methodological problem of whether the observer

Requests for reprints should be sent to S. C. Masin at Istituto di Psicologia, Piazza Capitaniato 3, 35139 Padova, Italy.

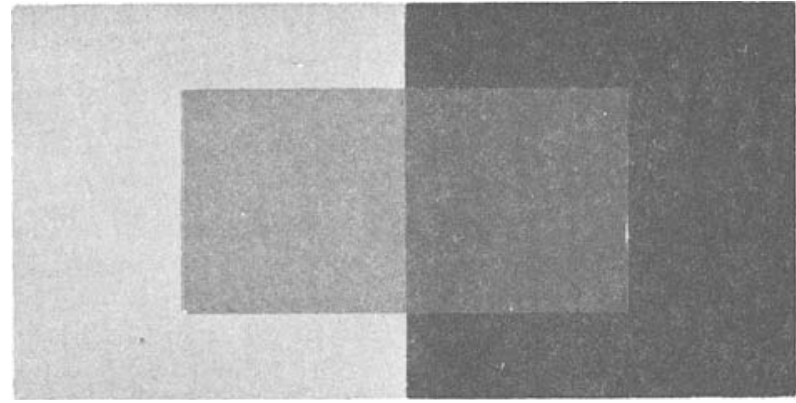

Figure 1. Example of a transparent surface on a bicolored background.

is able to produce an unbiased estimate of the reduction lightness under the analytical attitude. In a pattern such as that outlined in Figure $2 b$, in which regions $P$ and $Q$ are detached, the evaluation of the reduction lightness $p$ obviously cannot be influenced by the transparency phenomenon. That influence is possible only when the distance, 2d, between $P$ and $Q$ is nil. The existence of this influence may be checked by comparing the estimate of $p$ for $d \neq 0$ with the estimate of $p$ for $d=0$. More precisely, since $p$ must be some function of $d$, it may be concluded that there is such an influence only in the case in which the estimate of $p$ for $d=0$ deviates significantly from the curve relating $p$ to $d$.

\section{METHOD}

\section{Observers}

Fifty university students served as unpaid observers. They were recruited upon entering the institute.

\section{Stimuli}

Figure $2 b$ illustrates the patterns used in the experiment. The length of the side of the squares for background surfaces $A$ and $B$ was $40 \mathrm{~mm}$, and that for surfaces $P$ and $Q$ was $22 \mathrm{~mm}$. The distance $d$, indicated in Figure $2 b$, could assume one of four values: $0,1,4$, or $16 \mathrm{~mm}$. Two 

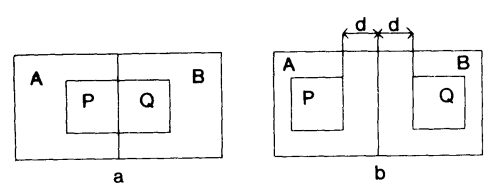

Figure 2. (a) Outline representation of the pattern shown in Figure 1. (b) Outline representation of one of the patterns used in this experiment. The distance $d$ was varied. The purpose of the experiment was to check whether the estimate of the reduction lightness of $P$ was the same when $d=0$ and $d \neq 0$.

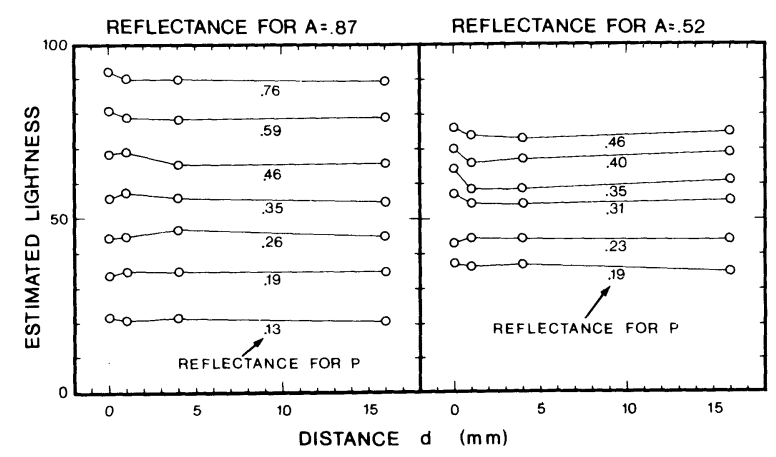

Figure 3. Mean estimate of lightness of surface $P$ in the experimental pattern as a function of the distance $d$ (Figure $2 b$ ), for each of the reflectances for surface $P$. The diagram on the left (right) refers to background surfaces $A$ and $B$ with reflectance $.87(.52)$ and .02 (.11), respectively.

bicolored backgrounds were used. The reflectances for A and B were, respectively, .87 and .02 , or .52 and .11 . Table 1 reports seven couples of reflectances for $\mathrm{P}$ and $\mathrm{Q}$, for each bicolored background. In all, there were 56 patterns, obtained by combining four distances $d$, two bicolored backgrounds, and seven couples of $\mathrm{P}$ and $\mathrm{Q}$. Transparency could be perceived only in 14 patterns, that is, in the ones for which $\mathrm{d}=0$. Surfaces A, B, P, and Q were made of gray paper. Papers from the set of 19 supplied by the NCS Institute (Sweden) were used. Each pattern was stuck on a $15 \times 15 \mathrm{~cm}$ white cardboard (reflectance of about .90-.95). Observers placed their heads on a chinrest to keep the eyes level with the stimulus. The pattern was placed in the frontal-parallel plane, and was $1.7 \mathrm{~m}$ distant from an observer's eyes. The illumination level was about $25 \mathrm{~lx}$.

\section{Procedure}

Each pattern was displayed, in a random order different for each observer, by removing a black screen. The observer was instructed to rate, within 5-10 sec, the achromatic color (lightness) of surface P (Figure 2b) using the numbers in the range $0-100$. He/she was told that the number 0 represented the blackest black ever experienced in his/her life, and 100 the whitest white. Other numbers represented intermediate grays. Observers were also allowed to use fractional numbers. Only integers were used, however. Each observer gave only one numerical response per pattern. A session lasted about $45 \mathrm{~min}$.

Table 1

Reflectances for Surfaces $A, B, P$, and $Q$ in the Patterns Used in this Experiment

\begin{tabular}{cccccccccc}
\hline $\begin{array}{c}\text { Background } \\
\text { Surface }\end{array}$ & $\begin{array}{c}\text { Reflec- } \\
\text { tance }\end{array}$ & Surface & \multicolumn{5}{c}{ Reflectance } \\
\hline A & .87 & P & .13 & .19 & .26 & .35 & .46 & .59 .76 \\
B & .02 & Q & .08 & .11 & .11 & .16 & .19 & .23 .26 \\
A & .52 & P & .19 & .23 & .23 & .31 & .35 & .40 .46 \\
B & .11 & Q & .16 & .16 & .19 & .23 & .23 & .23 .26 \\
\hline
\end{tabular}

\section{RESULTS AND CONCLUSION}

The results are represented in Figure 3. The mean rated lightness of $P$ is represented on the ordinate, and the distance $d$ is represented on the abscissa. The diagram on the left refers to the background reflectances .87 and .02 , and the diagram on the right to the background reflectances .52 and .11. The data points connected by a line correspond to a given reflectance for surface $P$. Each point represents the arithmetic mean of 50 single estimates. (As may be seen in Table 1, Row 3 , the reflectance .23 was used twice. The corresponding data points, in the diagram on the right in Figure 3, represent the mean of 50 means of the two single estimates.) A double standard error is roughly equal to the diameter of a circle.

An analysis of variance, in which the factors were the reflectance for $P$ and the distance $d$, was carried out separately for the data reported in the two diagrams. The results show that the main effects due to the factor "distance d" are not significant at the .05 level [left diagram: $\mathrm{F}(3,147)=.79$; right diagram: $\mathrm{F}(3,147)=2.18]$, and that the interaction also is not significant [left diagram: $\mathrm{F}(18,882)=.69$; right diagram: $\mathrm{F}(18,882)=1.38$ ].

These results show (1) that the observer is easily able to switch from the normal attitude (when the transparent surface is perceived as having a single homogeneous color, through which the color of the background is seen) to the analytical attitude (when the parts of the transparent surface are perceptually isolated), and (2) that there is, practically, no interference between these two modes of perceiving. Observers are therefore able to produce unbiased estimates of reduction lightness. It should be noted, however, that this conclusion applies only to the bidimensional patterns used in this experiment. There is still no proof that the normal and analytical attitudes do not interfere when the transparent surface is at some distance from the background.

\section{REFERENCES}

Beck, J., Prazdny, K., \& Ivry, R. (1984). The perception of transparency with achromatic colors. Perception \& Psychophysics, 35, 407-422.

FucHs, W. (1923). Experimentelle Untersuchungen über das simultane Hintereinandersehen auf derselben Sehrichtung. Zeitschrift für Psychologie, 91, 145-235.

Kanizsa, G. (1979). Organization in vision. New York: Praeger.

METELLI, F. (1982). Stimulation and perception of transparency (Report No. 73). Padua, Italy: University of Padua, Institute of Psychology.

Metelli, F. (1983). Stimolazione e percezione della trasparenza con tonalità acromatiche. Ricerche di Psicologia, 26, 159-180.

\section{NOTE}

1. Transparency may be disrupted in at least three independent ways: (1) by assuming an analytical attitude (Fuchs, 1923), (2) by altering the so-called topological or figural conditions of transparency (Kanizsa, 1979 ), or (3) by using an appropriate reduction screen. 